\title{
Mutations in the glucocerebrosidase gene are responsible for Chinese patients with Parkinson's disease
}

\author{
Zhe Yu ${ }^{1,5}$, Ting Wang ${ }^{1,5}$, Jun Xu ${ }^{1,2,5}$, Wei Wang ${ }^{1}$, Guifang Wang ${ }^{2}$, Chao Chen ${ }^{1}$, Lili Zheng ${ }^{1}$, Li Pan ${ }^{1}$, \\ Dianrong Gong ${ }^{2}$, Xueli $\mathrm{Li}^{2}$, Huaiqian $\mathrm{Qu}^{2}$, Fang $\mathrm{Li}^{3}$, Bin Zhang ${ }^{1}$, Weidong $\mathrm{Le}^{4}$ and Fabin Han ${ }^{1,2}$
}

Pathological mutations in the glucocerebrosidase gene (GBA) have been suggested to be associated with Parkinson's disease (PD) in various ethnic populations. Most studies on Chinese PD patients have only screened the N370S and L444P mutations in the GBA gene. To investigate the GBA mutations in Chinese population, we performed complete sequencing of the GBA gene in 184 Chinese PD patients and 130 Chinese control individuals. As a result, we identified three novel and nine reported GBA mutations. The novel mutations include 5-bp deletion (c.334_338deICAGAA), L264I and L314V and the nine reported GBA mutations are R163Q, F213I, E326K, S364S, F347L, V375L, L444P, RecNcil and Q497R. The novel 5-bp deletion (CAGAA) produces a short truncated GBA protein of 142 amino acids, which loses major function domains of the 536 amino acids. Our data also reveals that the frequency of GBA mutations within this Chinese PD cohort was $8.7 \%$, which is significantly higher than $1.54 \%$ observed in the Chinese control cohort $\left(\chi^{2}=7.22, P=0.0072\right.$; odds ratio $(O R)=6.095,95 \%$ confidence interval of $\mathrm{OR}=1.546-24.030$ ). The most common L444P mutation accounts $2.74 \%$, which confer more genetic risk for PD in this Chinese population. In conclusion, novel and known GBA mutations were identified and were found to be associated to PD in this Chinese population.

Journal of Human Genetics (2015) 60, 85-90; doi:10.1038/jhg.2014.110; published online 18 December 2014

\section{INTRODUCTION}

Parkinson's disease (PD) is the second most common neurodegenerative disease after Alzheimer's disease, affecting $1-2 \%$ of the population over the age of 60 . Most of the PD cases are sporadic and the rest are familial cases, which are caused by genetic mutations in PD-related genes. Over the last two decades, molecular studies have identified the genes of $\alpha$-synuclein (SNCA), Parkin (PARK2), DJ-1 (PARK7), PINK1, LRRK2, ATP13A2, VPS35 and EIF4G1 as being disease causing, whereas TAU(MAPT), NURR1(NR4A2), PARL and $G B A$ have been deemed as being susceptibility genes for PD. ${ }^{1-5}$ Mutations in the GBA gene (MIM 606463) were originally reported in patients with Gaucher's disease. Subsequently, GBA mutations were also identified in patients with Gaucher's disease and Parkinsonism. ${ }^{6}$

There has been an increasing evidence of the association between the GBA gene and PD in different ethnic populations and that the common GBA mutations in PD patients were reported to be N370S, L444P, RecNciI and D409H in the American, British, French, Greek,
Brazilian populations. The L444P and N370S were reported to account for $60-70 \%$ of the mutant alleles in some PD patients. ${ }^{7-9}$ Several studies performed screening for GBA L444P and N370S mutations in Chinese PD cases and controls and indicated that GBA L444P mutation is associated with $\mathrm{PD}$ in some Chinese populations. ${ }^{10-12}$ However, no studies have performed complete sequencing of the GBA gene for Chinese PD patients and controls. To characterize the GBA mutations and their association with PD in Chinese populations, we sequenced all exons of the GBA gene for PD patients and control individuals in a Chinese population.

\section{SUBJECTS AND METHODS}

Patients and control individual data

A total of 184 PD patients and 130 control individuals were recruited from two research hospitals in Eastern China. They include 74 patients from the Affiliated Liaocheng Hospital of Taishan Medical University (Liaocheng, Shandong, China) and 110 patients from Affiliated Reijing Hospital of Shanghai Jiaotong University (Shanghai, China). All these 184 PD patients are sporadic cases. The

${ }^{1}$ Centre for Stem cells and Regenerative Medicine, The Affiliated Liaocheng Hospital/Liaocheng People's Hospital, Taishan Medical University, Shandong, China; ${ }^{2}$ Department of Neurology, The Affiliated Liaocheng Hospital/Liaocheng People's Hospital, Taishan Medical University, Shandong, China; ${ }^{3}$ Department of Medicine, The Ottawa Hospital, University of Ottawa, Ontario, Canada and ${ }^{4}$ Institute of Neurology, Department of Neurology, The Affiliated Reijing Hospital, Shanghai Jiaotong University School of Medicine, Shanghai, China

${ }^{5}$ These authors contributed equally to this work.

Correspondence: Dr F Han, Centre for Stem Cells and Regenerative Medicine, Liaocheng People's Hospital/Affiliated Liaocheng Hospital, Taishan Medical University, 67 Dongchang West Road, Liaocheng, Shandong 252000, China.

E-mail: hanfabin2@gmail.com

or Dr W Le, Institute for Neurology, Department of Neurology, Affiliated Ruijin Hospital, Shanghai Jiaotong University, 197 Ruijin 2nd Road, Luwan District, Shanghai 200025, China. E-mail: wdle@sibs.ac.cn

Received 31 August 2014; revised 10 November 2014; accepted 18 November 2014; published online 18 December 2014 
diagnosis of PD and the exclusion criteria for secondary PD or another neurological disease were made on the criteria published by Hughs and Savitt. ${ }^{11,13}$ The age- and gender-matched 130 controls were collected from the same hospitals of PD cases. This study was approved by the Ethics Review Committees of Affiliated Liaocheng Hospital of Taishan Medical University and Affiliated Reijing Hospital of Shanghai Jiaotong University. Informed consents were obtained from all the PD patients and control individuals. Physical examinations were performed for all patients by two neurologists. The average age of the patients at onset of the PD was 60.89 years. The earliest age at onset was 36 years of age, whereas the latest age at onset occurred at 79 years of age in this Chinese population. The ratio of the men versus women in this PD cohort is 1.51 .

\section{DNA isolation, PCR amplification and direct sequencing of the GBA gene}

Genomic DNA was extracted from the venous blood of the patients and unaffected control individuals using the QiAamp Spin Column protocols of the QIAGEN kit (China-QIAGEN, Shanghai, China). Human GBA gene is located on chromosome 1q21 and includes 11 exons. In order to sequence only the $G B A$ gene and not the highly similar $G B A$ pseudogene (GBAP), we used three pairs of primers to specifically amplify three regions containing all of the exons in the GBA gene, with fragment 1 spanning exons $1-5$, fragment 2 spanning exons $5-7$ and fragment 3 spanning exons $8-11 .^{14}$ DNA sequencing was performed on ABI 3130xl genetic analyzer using BigDye Terminator v3.1 Cycle Sequencing Kit (Applied Biosystem, Foster City, CA, USA). The primer sequences for PCR amplification and DNA sequencing are available on ordering.

\section{Mutation confirmation and functional prediction of the identified mutations}

Single-nucleotide polymorphism (SNP) is generally defined as a common single-nucleotide variant that occurs in a significant proportion $(>1 \%)$ of a large population. Those variants that were not detected in 100 controls and the SNP database (dbSNP137) should be deemed as being mutations. In this study, even though 130 normal controls were sequenced, the identified GBA sequence variants in $\mathrm{PD}$ patients, which results in nonsynonymous amino-acid change or those occurring in splice sites were screened in additional 100 normal control individuals with matching ethnic backgrounds to further confirm whether the variants are mutations or not. For the potential damage of the identified mutations to GBA protein, we used polyphen2 (http://genetics.bwh.harvard. edu/pph2) program to perform the functional prediction of the identified mutations.

\section{Statistical analyses}

The GBA mutation frequencies in PD patients and control individuals from Chinese population were statistically analyzed by using the $\chi^{2-}$ test (SPSS statistics software 19.1, IBM Corporation, Armonk, NY, USA).

\section{RESULTS}

Mutations in the GBA gene identified in PD patients and control individuals

After sequencing all 11 exons of the GBA gene in 184 PD patients, we identified 12 different mutations including 3 novel and 9 known mutations among 16 of the $184 \mathrm{PD}$ patients of Chinese origin. We used NM_000157 as the GBA reference gene to compare the GBA sequencing data. The three novel identified mutations in $\mathrm{PD}$ patients are 5-bp deletion of c.334_338delCAGAA, L264I and L314V. We also detected the following nine known GBA mutations, R163Q, F213I, E326K, F347L, S364S, V375L, L444P, RecNciI (L444P+ A456P+ V460V) and Q497R within our PD patients. But we only found two mutations of F347L and A456P in the 130 control individuals we sequenced (Table 1 and Figure 1).

The most common GBA mutations in these Chinese PD patients are RecNciI including L444P, A456P and V460V (three patients), L444P (two patients) and L264I (two patients).

We compared the GBA mutation frequencies between PD patients and control individuals to determine whether GBA mutations are more frequent in PD patients than in control individuals. The GBA mutation frequency of $8.7 \%(16 / 184)$ in these Chinese PD patients is significantly $>1.54 \%(2 / 130)$ of the control individuals $\left(\chi^{2}=7.22\right.$, $P=0.0072$; odds ratio $=6.095,95 \%$ confidence interval of odds ratio $=1.546-24.030$; Table 1 ). Our study indicated that Chinese PD patients have 10 times more risk to have GBA mutation than normal controls, and $G B A$ mutations are associated with $\mathrm{PD}$, suggesting that the GBA gene is the most frequent genetic risk factor for PD patients in this Chinese population.

Table 1 Identified mutations in Chinese cohorts of PD patients and control individuals

\begin{tabular}{|c|c|c|c|c|c|c|c|c|}
\hline \multirow[b]{2}{*}{ GBA mutation } & \multirow[b]{2}{*}{ Mutation status } & \multirow[b]{2}{*}{$c D N A$} & \multirow[b]{2}{*}{ Protein } & \multirow[b]{2}{*}{ Exon } & \multicolumn{2}{|c|}{ PD patients (184) } & \multicolumn{2}{|c|}{ Controls (130) } \\
\hline & & & & & $\mathrm{N}$ & Frequency (\%) & $\mathrm{N}$ & Frequency (\%) \\
\hline del5 (CAGAA) & Novel & c.334-338 del5 & Truncated & 4 & 1 & 0.54 & 0 & 0.00 \\
\hline $\mathrm{R} 163 \mathrm{Q}$ & Known & c. $605 \mathrm{G}>\mathrm{A}$ & p.Arg163Gln & 6 & 1 & 0.54 & 0 & 0.00 \\
\hline F213I & Known & c. $754 \mathrm{~T}>\mathrm{A}$ & p.Phe213lle & 6 & 1 & 0.54 & 0 & 0.00 \\
\hline L264I & Novel & c. $907 \mathrm{C}>\mathrm{A}$ & p.Leu264lle & 7 & 2 & 1.09 & 0 & 0.00 \\
\hline L314V & Novel & c. $1057 C>G$ & p.Leu314Val & 8 & 1 & 0.54 & 0 & 0.00 \\
\hline E326K & Known & c. $1093 \mathrm{G}>\mathrm{A}$ & p.Glu326Lys & 8 & 1 & 0.54 & 0 & 0.00 \\
\hline F347L & Known & c. $1156 \mathrm{~T}>\mathrm{C}$ & p.Phe347Leu & 8 & 1 & 0.54 & 1 & 0.77 \\
\hline S364S & Known & c. $1209 \mathrm{C}>\mathrm{T}$ & p.Ser364Ser & 8 & 1 & 0.54 & 0 & 0.00 \\
\hline V375L & Known & c. $1240 G>C$ & p.Val375Leu & 9 & 1 & 0.54 & 0 & 0.00 \\
\hline L444P & Known & c. $1448 \mathrm{~T}>\mathrm{C}$ & p.Leu444Pro & 10 & 2 & 1.09 & 0 & 0.00 \\
\hline \multirow[t]{3}{*}{ RecNcil(L444P-A456P-V460V) } & Known & c. $1448 \mathrm{~T}>\mathrm{C}$ & p.Leu444Pro & 10 & 3 & 1.63 & 0 & 0.00 \\
\hline & & c. $1483 G>C$ & p.Ala456Pro & & 0 & 0.00 & 0 & 0.00 \\
\hline & & c. $1497 \mathrm{G}>\mathrm{C}$ & p.Val460Val & & 0 & 0.00 & 0 & 0.00 \\
\hline $\mathrm{A} 456 \mathrm{P}$ & Known & c. $1483 G>C$ & p.Ala456Pro & 10 & 0 & 0.00 & 1 & 0.77 \\
\hline Q497R & Known & c. $1607 A>G$ & p.GIn497Arg & 11 & 1 & 0.54 & 0 & 0.00 \\
\hline Total & & & & & 16 & 8.70 & 2 & 1.54 \\
\hline
\end{tabular}

Abbreviations: cDNA, complementary DNA; GBA, glucocerebrosidase gene; PD, Parkinson's disease. 
Novel GBA mutations

We identified three novel GBA mutations in 184 PD patients (Table 1 and Figure 1). One mutation, c.907C $>\mathrm{A}$, changes the amino acid of leucine to ileucine (L264I) in exon 7 and was found in two Chinese patients. Another novel mutation of c.1057C $>\mathrm{G}$ was found in exon 8 of the coding region in one Chinese patient. The mutation of c. $1057 \mathrm{C}>\mathrm{G}$ changes the amino acid of leucine to valine (L314V). For these three novel GBA mutations of 5-bp deletion, L264I, L314V, R163Q and F347L (we thought these two mutations are novel, but found they are known mutations later), we sequenced additional 100 normal control individuals without finding anyone having one of these five mutations. Thus, for each specific mutation of 5-bp deletion, L264I, L314V, R163Q and F347L, we totally sequenced 230 control individuals, but we only sequenced 130 control individuals for the whole gene. Importantly, we identified a novel mutation of 5-bp deletion (CAGAA) of exon 4 in one Chinese patient. This 5-bp deletion produces a frame-shift mutation leading to a truncated protein of 142 amino acids (Figure 2). After the 5-bp deletion was identified in the patient, we had tried to get the fresh blood and skin fibroblast from this patient to perform RT-PCR and western blotting analysis, unfortunately the patient passed away because of another disease. In order to know whether the identifed mutations have some

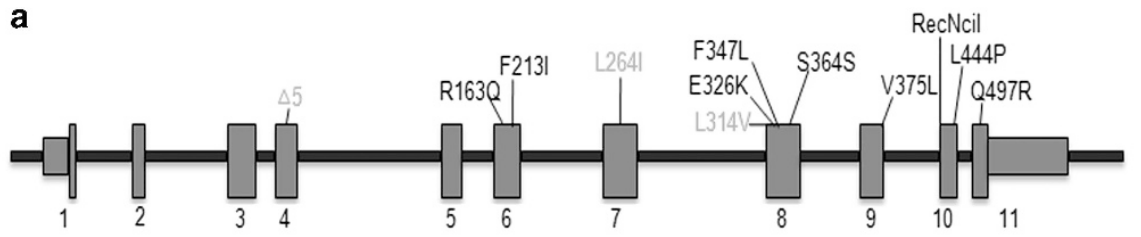

b $\triangle 5$ (CAGAA)

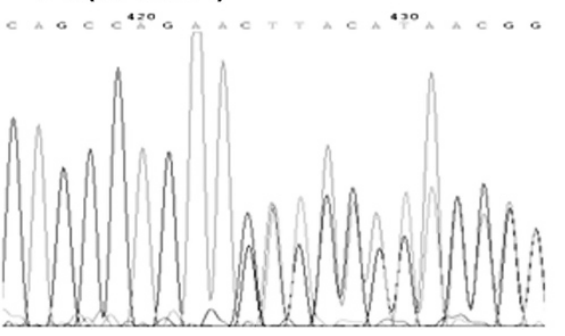

c

L264I

250

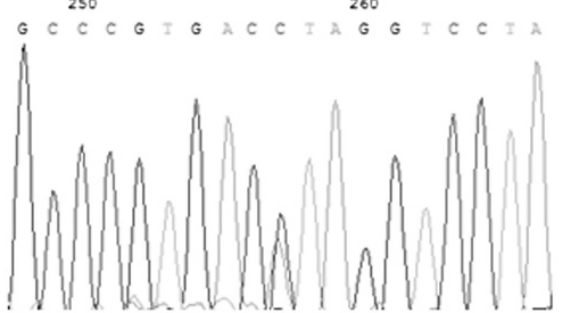

d

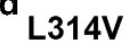

Figure 1 The identified glucocerebrosidase (GBA) mutations in Parkinson's disease (PD) patients from a Chinese population. (a) The exonic structure of GBA with 18 identified mutations, including 3 novel mutations and 9 known mutations. (b) Novel $\Delta 5$ represents 5-bp deletion of exon 4 in patient S25. (c) Novel L264I mutation in patients of L10 and L44. (d) Novel L314V mutation in patient S184. A full color version of this figure is available at the Journal of Human Genetics journal online.

MEFSSPSREECPKPLSRV SIMAGSLTGLLLLQAVSWASGARPCIPKSFGY S SVVCVCNAT 60
MEFSSPSREECPKPLSRV SIMAGSLTGLLLLQAVSWASGARPCIPKSFGYSSVVCVCNAT 60

YCDSFDPPTFPALGTFSRYESTR SGRRMELSMGPIQANHTGTGLLLTLQPE 111

YCDSFDPPTFPALGTFSRYESTRSGRRMELSMGPIQANHTGTGLLLTLQPE 111

VPESEGIWR GHDRCCCSQHPCPVTPCPKFAT* 142

QKFQKVKGF GGAMTDAAAL NILALSPPAQ NLLLKSYFSE EGIGYNIIRV PMASCDFSIR 170

TYTYADTPDDFQLHNFSLPE EDTKLKIPLI HRALQLAQRP VSLLASPWTS PTWLKTNGAV 230

NGKGSLKGQP GDIYHQTWAR YFVKFLDAYAEHKLQFWAVT AENEPSAGLL SGYPFQCLGF 290

TPEHQRDFIARDLGPTLANS THHNVRLLML DDQRLLLPHWAKVVLTDPEAAKYVHGIAVH 350

WYLDFLAPAKATLGETHRLF PNTMLFASEACVGSKFWEQS VRLGSWDRGM QYSHSITNL 410

LYHVVGWTDWNLALNPEGGP NWVRNFVD SP IIVDITKDTF YKQPMFYHLG HF SKFIPEGS 470

QRVGLVASQKNDLDAVALMH PDGSAVVVVL NRSSKDVPLT IKDPAVGFLE TISPGYSIHT 530

\section{YLWRRQ* 536}

Figure 2 The amino-acid sequence alignment of the truncated glucocerebrosidase (GBA) protein produced by the 5-bp deletion of the GBA gene and the wild-type GBA protein. The amino-acid sequence alignment of the truncated GBA protein produced by the 5-bp deletion of the GBA gene and the wild-type $G B A$ protein. This 5-bp deletion produces a frame-shift mutation leading to a truncated GBA protein of 142 amino acids, highlighted in red. The wild-type GBA protein of 536 amino acids is in black. A full color version of this figure is available at the Journal of Human Genetics journal online. 
pathological effects on the function of GBA protein, we used polyphen2 program (http://genetics.bwh.harvard.edu/pph2) to predict the protein damage of these novel and known mutations. As a result, we found that novel L264I mutation, known V375L and L444P mutations have possible damaging effects on GBA protein, whereas novel $\mathrm{L} 314 \mathrm{~V}$ and other known mutations are benign to GBA protein as shown in Table 2 .

As novel mutation of L264I (c.907C > A) was identified in two Chinese PD patients (L10 and L44), we performed haplotype analysis to know whether or not this mutation is from the same founder mutation using single-nucleotide polymorphism flanking this mutation. As a result, one PD patient has haplotype of CACC/GAAT (the third letter is $\mathrm{C}>\mathrm{A}$ mutation), whereas another patient has haplotype of CGCT/GGAT. No common haplotype was shared in these two patients, excluding this L264I mutation coming from the same founder.

Table 2 Predicted protein damage of the novel and known mutations identified in Chinese PD patients

\begin{tabular}{lllll}
\hline GBA Mutation $^{\text {a }}$ & Score & Sensitivity & Specificity & Effects \\
\hline R163Q & 0.012 & 0.96 & 0.78 & Benign \\
F213I & 0.117 & 0.93 & 0.86 & Benign \\
L264I & 0.613 & 0.87 & 0.91 & Possibly damaging \\
L314V & 0 & 1 & 0 & Benign \\
E326K & 0.015 & 0.96 & 0.79 & Benign \\
F347L & 0.002 & 0.99 & 0.3 & Benign \\
V375L & 0.963 & 0.78 & 0.95 & Probably damaging \\
L444P & 0.938 & 0.8 & 0.94 & Possibly damaging \\
A456P & 0.037 & 0.94 & 0.82 & Benign \\
Q497R & 0.001 & 0.99 & 0.15 & Benign \\
\hline
\end{tabular}

Abbreviation: GBA, glucocerebrosidase.

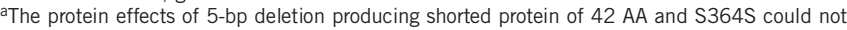
be predicted by polyphen 2 program.

\section{Mutation frequency of L444P and N370S}

Jewish PD patients were reported to have a high frequency of L444P and N370S accounting for $70 \%$ of PD cases among those with GBA mutation. ${ }^{7,8}$ Other ethnic PD patients were also found to have high frequency of L444P mutation. ${ }^{15,16}$ In 184 Chinese PD patients, we found two patients with the L444P mutation and three patients with the RecNciI mutation (L444P+ A456P+ V460V), but none of the Chinese PD patients had the N370S mutation. The mutation frequency of L444P in these Chinese PD patients was $2.72 \%(5 / 184)$. There were no mutations of N370S, L444P and RecNcil found in 130 control individuals. Overall, L444P represents $31.2 \%(5 / 16)$ of the GBA mutations in this Chinese PD cohort, which is similar to that of PD patients in other ethnic groups except for those of Jewish ancestry. ${ }^{17}$

Genotype-phenotype analyses of PD patients with $G B A$ mutations We analyzed the phenotype characteristics of PD patients with $G B A$ mutation and found that most of the PD patients with GBA mutations are L-DOPA responsive with motor fluctuations (Table 3). A previous study has suggested PD patients with GBA mutations had an earlier age of onset. ${ }^{17}$ We found that Chinese patients with $G B A$ mutations had an age at onset of 55.9 years, whereas patients without $G B A$ mutations had an age at onset of 61.6 years. However, no significant difference was observed between the age at onset of patients with GBA mutations and without $G B A$ mutations $(t=0.412, P=0.522)$, probably because the samples with $G B A$ mutations are too small. The patients with the RecNciI mutation were also found to have more severe clinical symptoms of tremor and slow movement. We found that most of the PD populations have GBA mutation frequencies in the range of $4-10 \%$ except for the Jewish patients who have a higher frequency of $13-20 \%{ }^{7,8,18,19}$

\section{DISCUSSION}

Recent studies have reported mutations in GBA for PD from different ethnic origins and revealed that $G B A$ mutations have conferred more

Table 3 Clinical phenotype characteristics of Chinese PD patients with GBA mutations

\begin{tabular}{|c|c|c|c|c|c|c|c|c|}
\hline $\begin{array}{l}\text { Patient } \\
\text { number }\end{array}$ & Mutations & $\begin{array}{l}\text { Clinical } \\
\text { diagnosis }\end{array}$ & Sex & $\begin{array}{c}\text { Age at onset } \\
\text { (years) }\end{array}$ & $\begin{array}{l}\text { L-DOPA } \\
\text { responsiveness }\end{array}$ & $\begin{array}{c}\text { Family history } \\
\text { Number of patients }\end{array}$ & First symptom & $\begin{array}{l}\text { Cognitive } \\
\text { symptoms }\end{array}$ \\
\hline L10 & L264I & PD definite & Female & 60 & Yes & No & Stiffness in the right arm & No \\
\hline L14 & E326K & Parkinsonism & Male & 67 & Yes & No & Tremor in the legs & No \\
\hline L15 & S364S & Parkinsonism & Male & 49 & Yes & No & Tremor in the left leg & No \\
\hline L17 & RecNcil & PD definite & Female & 38 & Yes & No & Stiffness in the left arm & No \\
\hline L33 & F347L & PD definite & Male & 58 & Yes & No & Slow movement in the right arm & No \\
\hline L43 & RecNcil & PD definite & Female & 54 & Yes & No & Stiffness in the legs & Yes \\
\hline L44 & L264I & PD definite & Male & 63 & Yes & No & Cognitive impairment & Yes \\
\hline L75 & RecNcil & PD definite & Female & 46 & yes & No & Stiffness in the legs & No \\
\hline S25 & $\begin{array}{c}\text { del5 } \\
\text { (CAGAA) }\end{array}$ & PD definite & Male & 49 & Yes & No & Tremor on the left side & No \\
\hline S47 & L444P & PD definite & Male & 56 & Yes & No & Tremor in the arms & No \\
\hline $\mathrm{S} 50$ & V375L & PD definite & Male & 59 & Yes & No & Stiffness in the right larm & No \\
\hline S51 & L444P & PD definite & Male & 63 & Yes & No & Stiffness in the legs & No \\
\hline S157 & Q497R & PD definite & Female & 65 & Yes & No & Tremor in the right leg & Yes \\
\hline S159 & F213I & PD definite & Female & 51 & Yes & No & Slow movement in the left arm & No \\
\hline S184 & L314V & PD definite & Female & 61 & Yes & No & Tremor and stiffness in the legs & No \\
\hline S195 & $\mathrm{R} 163 \mathrm{Q}$ & PD definite & Female & 56 & Yes & No & Tremor in the arms & No \\
\hline Average & & & & 55.94 & & & & \\
\hline
\end{tabular}

Abbreviations: GBA, glucocerebrosidase; PD, Parkinson's disease. 
risk to the development of $\mathrm{PD}$ than any of other PD-related genes such as LRRK2 and Parkin (PARK2). However, most of these studies focused on the specific $G B A$ mutations without sequencing the full $G B A$ gene. By complete sequencing analyses of the GBA gene, we found that PD patients have a significantly higher frequency of $G B A$ mutations than their corresponding control individuals from a Chinese population. The GBA mutation frequency $(8.7 \%)$ in this Chinese cohort of PD patients is comparable to the reported GBA mutation frequencies in $\mathrm{PD}$ patients with different ethnic backgrounds in other studies. ${ }^{7,8,15}$ Our study provided further evidence supporting the idea that the overall frequency of GBA mutations within PD patients might not be affected by ethnic origin except for the Jewish population. 5

The L444P mutation was reported to be the most frequent GBA mutation in PD populations occurring at 1.14\%, 2.00\% and 1.39\% incidence among the Canadian, American non-Jewish and British patients, respectively. ${ }^{15,17,20}$ Sun et al. ${ }^{10}$ screened 402 Chinese PD patients and reported that the L444P allele frequency was $2.74 \%$ (11/402). Mao et al. ${ }^{11}$ screened L444P mutation in 616 Chinese PD patients and found that the frequency of this L444P mutation is 3.2\% (20/616), which is similar than $2.74 \%$ in our Chinese PD patients. Even though Ashkenazi Jewish and some European PD patients were reported to have the N370S allele frequencies of 15 and $46 \%,{ }^{7,8}$ the N370S mutation frequency is relatively lower in Asian PD patients. ${ }^{9,21,22}$ We did not identify the N370S mutation in any of the 184 Chinese PD patients, further supporting that N370S mutation occurred rarely in Asian PD patients.

We only saw a few patients with cognitive impairment and dementia, and did not see an increased risk of developing cognitive impairment and dementia symptoms in patients with GBA mutations, a result which differs from others. ${ }^{23,24}$ Even though the patients with $G B A$ mutations were reported to have bradykinesia and rigidity as the earlier symptom, ${ }^{25}$ the earlier symptoms of our PD patients with GBA mutations included tremors, rigidity and cognitive impairment and vary from one to another. We could not find any difference in the motor symptoms, cognitive impairment or disease progression between the $G B A$ mutation carriers and non-GBA mutation carriers.

We have identified 3 novel GBA mutations in 184 Chinese PD patients, suggesting that the screening of GBA mutation in a larger cohort of Chinese PD patients would further help understand the role $G B A$ has in the pathogenesis of PD. One of the novel GBA mutations (5-bp deletion) was identified in a Chinese PD patient. As this 5-bp deletion produces a short truncated GBA protein, potentially resulting in a more severe pathology, we further evaluated the clinical characteristics of the patient. This patient developed a tremor on the left side and slow movement as the first symptoms at the age of 49 . $\mathrm{He}$ is L-DOPA-responsive but does not have other cognitive symptoms. The L264I mutation was found in two sporadic Chinese PD patients, but haplotype analysis showed that they do not share the same haplotype, suggesting that this mutation does not occur as linkage disequilibrium in this Chinese population.

GBA mutation in patients with Lewy body Dementia, Alzheimer's disease and multiple system atrophy ${ }^{17,26,27}$ were ever examined to determine whether GBA mutations contributed to pathogenesis of other neurodegenerative diseases. As a result, GBA mutations were also identified in Lewy body Dementia and Alzheimer's disease patients, but not in the multiple system atrophy patients, suggesting that the GBA mutations could induce neurodegeneration in other neurological disorders through mechanisms similar to PD. ${ }^{28}$

The mechanism whereby GBA mutations lead to the death of dopaminergic neurons is unknown. In most patients with $G B A$ mutations, $\beta$-glucosidase activity was not affected, suggesting that the $G B A$ mutations may affect the dopamine neuron survival through other mechanisms. Some studies showed that mutant GBA affects dopamine neuron development and maturation by interfering with $\alpha$-synuclein in the mitochondria and protein trafficking signaling of the dopamine neurons. ${ }^{12} \mathrm{~A}$ recent study investigating the overexpression of the GBA mutants (L444P, N370, D409H, D409V, E235A and $\mathrm{E} 340 \mathrm{~A}$ ) showed a significant increase in the levels of $\alpha$-synuclein in cultured human and mouse neural cells. ${ }^{29}$ Another study suggested that the GBA mutations could interfere with the endoplasm reticulumassociated degradation of the proteins to induce cell death. ${ }^{30}$ The prediction analysis on functional effects of these novel and known mutations suggested that L264I, V375L and L444P are possibly damaging the GBA protein, whereas others may not have pathological effects on the GBA protein. To further validate the damaging effects of L264I, V375L and L444P mutations more studies are required to overexpress these mutations in cell cultures and in gene-targeted animal models to understand the molecular pathways these mutations are involved in. In conclusion, this study indicates that GBA mutations are the risk factor in Chinese PD patients.

\section{CONFLICT OF INTEREST}

The authors declare no conflict of interest.

\section{ACKNOWLEDGEMENTS}

We thank all the participants from Canada and China. Funding was provided by the research grants from Research scholar Fund of Liaocheng People's Hospital of Shandong province (NO. 2011LCYYF001), China, National Natural Science Foundation of China (NSFC 81271251). We thank Ruobing Zou for preparing blood samples from Canada. Statistical analysis was performed by Professor Yongjun Zhao, Department of Health Statistics, Binzhou Medical College of China. This study was supported by the research grants from National Natural Science Foundation of China (NSFC 81271251).

Author contributions: Dr Fabin Han contributed to the research design, clinical diagnosis of PD patients, some experimental work and manuscript preparation; Dr Weidong Le contributed to the research design, clinical diagnosis of PD patients and manuscript corrections; Zhe Yu, Ting Wang, Dr Jun Xu, Wang Wang, Chao Chen, Lili Zheng, Li Pan, Fang Li, Lemuel Racacho contributed to the experimental work; Dr Bin Zhang, Dr Dianrong Gong, Dr Huaiqian Qu, Dr Guifang Wang, Dr Xueli Li and Dr Jun Xu contributed to clinical diagnosis of PD patients.

1 Zimprich, A., Benet-Pages, A., Struhal, W., Graf, E., Eck, S. H. \& Offman, M. N. et al. A mutation in VPS35, encoding a subunit of the retromer complex, causes late-onset Parkinson disease. Am. J. Hum. Genet. 89, 168-175 (2011)

2 Lill, C. M., Roehr, J. T., McQueen, M. B., Kavvoura, F. K., Bagade, S. \& Schjeide, B. M. et al. Comprehensive research synopsis and systematic meta-analyses in Parkinson's disease genetics: the PDGene database. PLoS Genet. 8, e1002548 (2012).

3 Gasser, T. Molecular pathogenesis of Parkinson disease: insights from genetic studies. Expert Rev. Mol. Med. 11, e22 (2009).

4 Grimes, D. A., Han, F., Panisset, M., Racacho, L., Xiao, F. \& Zou, R. et al. Translated mutation in the Nurr1 gene as a cause for Parkinson's disease. Mov. Disord. 21, 906-909 (2006)

5 Beavan, M. S. \& Schapira, A. H. Glucocerebrosidase mutations and the pathogenesis of Parkinson disease. Ann. Med. 45, 511-521 (2013).

6 Lwin, A., Orvisky, E., Goker-Alpan, O., LaMarca, M. E. \& Sidransky, E. Glucocerebrosidase mutations in subjects with parkinsonism. Mol. Genet. Metab. 81, 70-73 (2004).

7 Lesage, S., Anheim, M., Condroyer, C., Pollak, P., Durif, F. \& Dupuits, C. et al. Largescale screening of the Gaucher's disease-related glucocerebrosidase gene in Europeans with Parkinson's disease. Hum. Mol. Genet. 20, 202-210 (2011).

8 Sidransky, E., Nalls, M. A. Aasly, J. O., Aharon-Peretz, J., Annesi, G. \& Barbosa, E. R. et al. Multicenter analysis of glucocerebrosidase mutations in Parkinson's disease. N. Engl. J. Med. 361, 1651-1661 (2009).

$9 \mathrm{Hu}$, F. Y., Xi, J., Guo, J., Yu, L. H., Liu, L. \& He, X. H. et al. Association of the glucocerebrosidase N370S allele with Parkinson's disease in two separate Chinese Han populations of mainland China. Eur. J. Neurol. 17, 1476-1478 (2010). 
10 Sun, Q. Y., Guo, J. F., Wang, L., Yu, R. H., Zuo, X. \& Yao, L. Y. et al. Glucocerebrosidase gene L444P mutation is a risk factor for Parkinson's disease in Chinese population. Mov. Disord. 25, 1005-1011 (2010).

11 Mao, X. Y., Burgunder, J. M., Zhang, Z. J., An, X. K., Zhang, J. H. \& Yang, Y. et al. Association between GBA L444P mutation and sporadic Parkinson's disease from Mainland China. Neurosci. Lett. 469, 256-259 (2010).

12 Wang, Y., Liu, L., Xiong, J., Zhang, X., Chen, Z. \& Yu, L. et al. Glucocerebrosidase L444P mutation confers genetic risk for Parkinson's disease in central China. Behav. Brain. Funct. 8, 57 (2012).

13 Hughes, A. J., Daniel, S. E. \& Lees, A. J. Improved accuracy of clinical diagnosis of Lewy body Parkinson's disease. Neurology 57, 1497-1499 (2001).

14 Mitsui, J., Mizuta, I., Toyoda, A., Ashida, R., Takahashi, Y. \& Goto, J. et al. Mutations for Gaucher disease confer high susceptibility to Parkinson disease. Arch. Neurol. 66, 571-576 (2009).

15 Neumann, J., Bras, J., Deas, E., O'Sullivan, S. S., Parkkinen, L. \& Lachmann, R. H et al. Glucocerebrosidase mutations in clinical and pathologically proven Parkinson's disease. Brain 132, 1783-1794 (2009).

16 De Marco, E. V., Annesi, G., Tarantino, P., Rocca, F. E., Provenzano, G. \& Civitelli, D. et al. Glucocerebrosidase gene mutations are associated with Parkinson's disease in southern Italy. Mov. Disord. 23, 460-463 (2008).

17 Clark, L. N., Ross, B. M., Wang, Y., Mejia-Santana, H., Harris, J. \& Louis, E. D. et al. Mutations in the glucocerebrosidase gene are associated with early-onset Parkinson disease. Neurology 69, 1270-1277 (2007).

18 Bras, J., Paisan-Ruiz, C., Guerreiro, R., Ribeiro, M. H., Morgadinho, A. \& Januario, C. et al. Complete screening for glucocerebrosidase mutations in Parkinson disease patients from Portugal. Neurobiol. Aging 30, 1515-1517 (2009).

19 Kalinderi, K., Bostantjopoulou, S., Paisan-Ruiz, C., Katsarou, Z., Hardy, J. \& Fidani, L. Complete screening for glucocerebrosidase mutations in Parkinson disease patients from Greece. Neurosci. Lett. 452, 87-89 (2009).

20 Sato, C., Morgan, A., Lang, A. E., Salehi-Rad, S., Kawarai, T. \& Meng, Y. et al. Analysis of the glucocerebrosidase gene in Parkinson's disease. Mov. Disord. 20, 367-370 (2005).
21 Tan, E. K., Tong, J., Fook-Chong, S., Yih, Y., Wong, M. C. \& Pavanni, R. et al. Glucocerebrosidase mutations and risk of Parkinson disease in Chinese patients. Arch. Neurol. 64, 1056-1058 (2007).

22 Ziegler, S. G., Eblan, M. J., Gutti, U., Hruska, K. S., Stubblefield, B. K. \& Goker-Alpan, 0 . et al. Glucocerebrosidase mutations in Chinese subjects from Taiwan with sporadic Parkinson disease. Mol. Genet. Metab. 91, 195-200 (2007).

23 Goker-Alpan, O., Lopez, G., Vithayathil, J., Davis, J., Hallett, M. \& Sidransky, E. The spectrum of parkinsonian manifestations associated with glucocerebrosidase mutations. Arch. Neurol. 65, 1353-1357 (2008).

24 Gan-Or, Z., Giladi, N. \& Orr-Urtreger, A. Differential phenotype in Parkinson's disease patients with severe versus mild GBA mutations. Brain 132, e125 (2009).

25 Eblan, M. J., Nguyen, J., Ziegler, S. G., Lwin, A., Hanson, M. \& Gallardo, M. et al. Glucocerebrosidase mutations are also found in subjects with early-onset parkinsonism from Venezuela. Mov. Disord. 21, 282-283 (2006).

26 Segarane, B., Li, A., Paudel, R., Scholz, S., Neumann, J. \& Lees, A. et al. Glucocerebrosidase mutations in 108 neuropathologically confirmed cases of multiple system atrophy. Neurology 72, 1185-1186 (2009).

27 Farrer, M. J., Williams, L. N., Algom, A. A., Kachergus, J., Hulihan, M. M. \& Ross, O. A et al. Glucosidase-beta variations and Lewy body disorders. Parkinsonism Relat. Disord. 15, 414-416 (2009).

28 Tsuang, D., Leverenz, J. B., Lopez, O. L., Hamilton, R. L., Bennett, D. A. \& Schneider, J. A. et al. GBA mutations increase risk for Lewy body disease with and without Alzheimer disease pathology. Neurology 79, 1944-1950 (2012).

29 Cullen, V., Sardi, S. P., Ng, J., Xu, Y. H., Sun, Y. \& Tomlinson, J. J. et al Acid beta-glucosidase mutants linked to Gaucher disease, Parkinson disease, and Lewy body dementia alter alpha-synuclein processing. Ann. Neurol. 69, 940-953 (2011).

30 Ron, I. \& Horowitz, M. ER retention and degradation as the molecular basis underlying Gaucher disease heterogeneity. Hum. Mol. Genet. 14, 2387-2398 (2005). 